\title{
Sweden leads EU in developing new antibiotics
}

$\mathrm{L}$ inus Sandegren's hunt for new antibiotic drugs started with a shock. As a drug researcher in Uppsala, a small city in southern Sweden, Sandegren witnessed northern Europe's first outbreak of extended spectrum beta lactamase (ESBL) producing Enterobacteriaceae, which is resistant to all commonly used antibiotics. It hit the city's main hospital in 2005; conventional antibiotic therapies failed and 248 patients required complex, expensive care. The event was a harbinger: two years later, 2099 ESBL cases were reported in Sweden. By 2012, that figure had more than tripled.

"Antibiotic resistance has quickly become a severe menace," says Sandegren. The outbreak led him to join an integrated effort to develop new antibiotic drugs at Uppsala University's Department of Medical Biochemistry and Microbiology. "Unless new drugs are developed, things as common as a child's strep throat could kill again."

In Europe, antimicrobial resistance is now estimated to cause 25000 deaths annually and cost more than $€ 1.5$ billion ( $\$ 2.2$ billion) in health care expenses and productivity losses. However, since 1990, the number of large and mid-sized pharmaceutical companies developing antibiotics has dropped from 18 to just 5 , states a 2013 report by the United Kingdom Chief Medical Officer.

Since the 2005 outbreak, new antibiotic drug development has become a preoccupation not just at Uppsala, but in Sweden as a whole.

A new drug research paradigm is emerging, says Katarina Bjelke, director-general of researach policy at the Swedish Ministry of Education and Research. "The pharmaceutical industry is ... collaborating with universities much more to do the early phases of drug development."

To support this work, the Swedish government announced in 2013 that it would double its investment in life science through to 2016 from $€ 15$ million ( $\$ 22$ million) to $€ 33$ million (\$48 million).

the most promising aims to develop an ESBL antibody from chicken eggs.

Sweden's leadership in antibiotic control began in 1986, when it was the first country to severely restrict antibiotic use in meat production. In 1994, the increase in antibiotic use and resistance triggered the creation of the Swedish Strategic Programme for the Rational Use of Antimicrobial Agents and Surveillance of Resistance (Strama). The national focus on antibiotic control soon paid off: between 1995 and 2004, antibiotic use for outpatients decreased almost $25 \%$.

While tackling antibiotic control, the Swedish government began pushing for new drug development, explains Strama's chairman and Uppsala University infectious diseases researcher Otto Cars.

When Sweden's turn came to hold the Presidency of the European Union (EU) in 2009 , it aggressively promoted the launch of a massive research program targeting new antibiotic development,

"We want a chain from basic research into clinical trials into product development," says Bjelke. "The decision is to put money into this area where we have strong links. We don't want to see those links broken."

Erik Forsberg, managing director of Uppsala Bio, a government-funded life sciences incubator that seeds drug development ventures, says almost all drug research revolves around the university and a cluster of startup companies pursuing experimental innovations.

Like Sandegren and Bjelke, Forsberg sees new antibiotic drug development as a shrewd commercial and medical strategy. In recent years, Forsberg has assessed more than 250 related proposals and backed 18 ideas with grants ranging up to a million dollars. One of says Cars. Since 2007, the EU has invested nearly $\$ 1$ billion in this area, including \$140 million in funding for 15 new research initiatives announced last November. These initiatives include a research project focused on developing a new streptococcal vaccine, and another on developing nanoformulated antimicrobial peptides to treat bacterial infectious disease.

With only a fiftieth of Europe's population, Sweden's role in new antibiotic development is a source of national pride, says Cars. "You have to give credit to the Swedish government for initiating it and pushing the EU to deliver." - Paul Christopher Webster, Uppsala, Sweden

CMAJ 2014. DOI:10.1503/cmaj.109-4694 